\title{
Características productivas y textiles de la fibra en alpacas Huacaya de Puno, Perú
}

\author{
Productive and textile characteristics of fibre in Huacaya alpacas from Puno, Perú \\ Lorenzo A. Roque Gonzales ${ }^{1}$, Edwin Ormachea Valdez ${ }^{2,3}$
}

\section{Resumen}

El estudio tuvo como objetivo determinar las características productivas y textiles de la fibra en alpacas Huacaya considerando la edad (2, 4 y 6 años), sexo y procedencia. Se tomaron muestras de fibra del costillar medio de 120 alpacas de dos comunidades del distrito de Ayaviri, Puno. Se determinó el díametro, factor de confort, índice de curvatura y la finura al hilado utilizando el equipo Análisis Óptico del Diámetro de Fibra 2000 y la longitud de fibra mediante el método de la ASTM. El diámetro de fibra se incrementó y la finura al hilado se afectó significativamente con la edad $(\mathrm{p}<0.05)$, en tanto que el índice de confort y la longitud de mecha disminuyeron significativamente con la edad del animal $(\mathrm{p}<0.05)$. El índice de curvatura no se afectó con la edad, sexo o procedencia del animal. El sexo y la comunidad de procedencia no afectaron el diámetro de fibra el índice de confort, la longitud de mecha ni la finura al hilado. Se encontró una correlación negativa y moderada entre el diámetro de fibra con el índice de curvatura $(\mathrm{r}=-0.40)$ y factor de confort $(\mathrm{r}=-0.58)$, en tanto que se observó una correlación alta entre diámetro de fibra y finura al hilado $(\mathrm{r}=0.75)$.

Palabras clave: alpaca; diámetro de fibra; factor de picazón; índice de curvatura; longitud de mecha

\section{Abstract}

The objective of this study was to determine the productive and textile characteristics of the fibre in Huacaya alpacas considering age (2, 4 and 6 years), sex and origin. Fibre samples were collected from the flanks of 120 alpacas from two communities of the Ayaviri district, Puno. The diameter, comfort factor and curvature index and fineness of

\footnotetext{
${ }^{1}$ Escuela de Post Grado, Universidad Nacional del Altiplano, Puno, Perú

2 Departamento Académico de Medicina Veterinaria y Zootecnia, Universidad Nacional Amazónica, Madre de Dios, Perú

${ }^{3}$ E-mail: edwinormachea@gmail.com
}

Recibido: 7 de enero de 2018

Aceptado para publicación: 20 de junio de 2018 
yarn was determined using the computer analysis Optical Fiber Diameter 2000 and fibre length using the ASTM method. The diameter of the fibre and the fineness of yarn increased significantly with age $(\mathrm{p}<0.05)$, while the comfort index and wick length decreased significantly with the age of the animal $(\mathrm{p}<0.05)$. The curvature index was not affected by age, sex or origin of the animal. The sex and the community of origin did not affect the fibre diameter, the comfort index, the length of the wick or the fineness of the yarn. A negative and moderate correlation was found between the diameter of fibre with the index of curvature $(\mathrm{r}=-0.40)$ and comfort factor $(\mathrm{r}=-0.58)$, while a high correlation was observed between fibre diameter and fineness of yarn $(\mathrm{r}=0.75)$.

Key words: alpaca; fibre diameter; itching factor; curvature index; staple length

\section{INTRODUCCIÓN}

La población de alpacas en el Perú es aproximadamente de 3685500 , donde, la raza Huacaya representa el $80.4 \%$, la raza Suri con $12.2 \%$ y los cruces con $7.3 \%$ (INEI, 2012). La producción alpaquera se encuentra principalmente en las zonas altoandinas de Puno, Cusco, Huancavelica, Arequipa, Apurímac y Ayacucho (Benavides et al., 1996).

El Perú es considerado como el principal productor de fibra de alpaca en el mundo. El 85\% de la producción nacional está orientada al mercado internacional y representa el $1.35 \%$ de las exportaciones (FAO, 2005). En la actualidad, la producción ha ido disminuyendo debido a la variabilidad en cuanto a finura en el rebaño y uno de los inconvenientes de la fibra es la falta de uniformidad en el diámetro a lo largo de su longitud, la misma que repercute en la calidad de la fibra (Wuliji et al., 2000; Quispe et al., 2008). El diámetro de fibra, el peso de vellón, el factor de confort y la finura al hilado son las principales características desde el punto de vista comercial y manufacturero (Quispe et al., 2009a).

Bajo esta perspectiva, se realizó el trabajo de investigación con el propósito de establecer información objetiva para el inicio de un programa de mejoramiento genético en alpacas, en relación a las características pro- ductivas textiles de la fibra en alpacas provenientes de dos comunidades del distrito de Ayaviri, Melgar, Perú.

\section{Materiales y Métodos}

El trabajo de investigación se realizó en el distrito de Ayaviri, provincia de Melgar, en Puno, Perú. La zona se encuentra a una altitud $4370 \mathrm{~m}$. El clima es seco y frío, con dos épocas bien definidas, una de estiaje (abril a setiembre) y otra de lluvias (octubre a marzo). Se trabajó con 120 alpacas Huacaya de tres edades (2, 4 y 6 años), hembras y machos.

El método de muestreo utilizado en el estudio fue no probabilístico del tipo aleatorio simple. Para ello se trabajó con 120 alpacas Huacaya de color blanco. La edad de los animales se determinó utilizando registros de producción de los propietarios y se trabajó con animales de tres edades (2, 4 y 6 años). Así mismo, se consideró el sexo (hembra, macho) y la procedencia de los animales (comunidades de Condormilla Alto [Huarachani] y Condormilla bajo [Quinsapugio]).

La toma de muestras de fibra se realizó en los meses de octubre a noviembre antes de la esquila. Se cortaron mechas de fibras (4 g) de la región del costillar medio como indica Aylan-Parker y McGregor (2001). Las muestras fueron colocadas en bolsas de 
polietileno rotuladas con el nombre del propietario, comunidad, edad y sexo del animal. El análisis se realizó en el laboratorio de fibras de la Estación Experimental Maranganí de la Universidad Nacional Mayor de San Marcos, en Cusco, Perú. Se determinó el diámetro, finura al hilado, factor de confort e índice de curvatura con el equipo Optical Fiber Diameter Análisis 2000, siguiendo las recomendaciones de Brims et al. (1999), y la longitud de fibra mediante el método de la ASTM (ASTM, 1999), Designación D 1234-85 (Cordero, 2011).

El efecto de la edad, sexo y comunidad sobre las variables en estudio se calcularon utilizando la aplicación R v. 3.0.2 (R Core Team, 2014).

\section{Resultados y Discusión}

\section{Diámetro de Fibra}

El diámetro de fibra aumentó significativamente con la edad $(\mathrm{p}<0.05)$, no habiendo diferencia por efecto del sexo o procedencia (Cuadro 1). Los valores obtenidos presentan grandes variaciones, especialmente con la edad, tal y como lo reportan otros autores (Huamaní y Gonzáles, 2004; Lupton y Stobart, 2006; McGregor, 2006; Diaz et al., 2015; Ormachea et al., 2015; Machaca et al., 2017).

Los resultados encontrados son inferiores a lo reportado por Holt (2006) en Australia quien obtuvo valores de 24.26, $25.78 \mathrm{y}$ $27.02 \mu \mathrm{m}$ en alpacas de 2,4 y 6 años, respectivamente, y a los valores de 24.6, 25.57 y 26.74 ìm en alpacas de 2, 3 y 4 años reportados por Huamaní y González (2004) en Huancavelica, Perú; diferencias que podrían deberse a factores genéticos, tamaño de muestra utilizada y al medio ecológico. Las variaciones también pueden ser debidas al factor alimentación, ya que juega un rol muy importante en la determinación del diámetro de fibra. Franco et al. (2009) menciona que niveles alimenticios bajos en energía y proteína disminuyen el diámetro de fibra, longitud y volumen.

Aylan-Parker y McGregor (2002) y Lupton y Stobart (2006) indican que las hembras tienen menor finura de fibra por tener requerimientos nutricionales más altos, debido a la lactación y y la gestación. Por otro lado, otros autores (Montes et al., 2008; Morante et al., 2009; Quispe et al., 2009b) señalan que los machos tienen fibras más finas que las hembras debido a una mayor presión de selección en los machos. No obstante, los resultados del presente estudio indican que no hubo diferencia en la finura por efecto del sexo, posiblemente debido a que los productores de alpaca de las dos comunidades en estudio no realizan una selección rigurosa de machos. Así mismo, los resultados concuerdan con diversos autores (Wuliji et al., 2000; McGregor y Butler, 2004; Diaz et al., 2015; Ormachea et al., 2015; Machaca et al., 2017).

Huanca et al. (2007) reporta diámetros de fibra de $22.71 \mu \mathrm{m}$ para la comunidad de Cojata (puna húmeda) y de $22.79 \mu \mathrm{m}$ para Santa Rosa (puna seca), valores inferiores a los obtenidos en este estudio.

\section{Factor de Confort}

El factor de confort disminuyó significativamente con la edad $(\mathrm{p}<0.05)$, no habiendo diferencia por efecto del sexo o procedencia (Cuadro 2).

Estos resultados concuerdan con hallazgos de otros autores (Lupton y Stobart, 2006; McGregor, 2006; Ormachea et al., 2015). Los resultados de este estudio presentan mejores valores de confort que los obtenidos por Lupton y Stobart (2006) de 82.7, 74.7 y $58.6 \%$ para alpacas de 1, 2 y más de 3 años, posiblemente debido a que dichos animales presentaban un mayor diámetro de fibra. Por otro lado, Quispe et al. (2009a) en estudios realizados en alpacas provenientes de ocho comunidades de la región de Huancavelica 
Cuadro 1. Diámetro $(\mu \mathrm{m})$ de fibra en alpacas Huacaya, según edad, sexo y procedencia

\begin{tabular}{lcccc}
\hline Variable & N. $^{\circ}$ & Media \pm D.E. & Máximo & Mínimo \\
\hline Edad (años) & & & & \\
2 & 40 & $21.22 \pm 1.79^{\text {a }}$ & 24.78 & 18.12 \\
4 & 40 & $23.35 \pm 1.98^{\mathrm{b}}$ & 28.19 & 20.15 \\
6 & 40 & $25.48 \pm 2.27^{\mathrm{c}}$ & 29.67 & 20.05 \\
Sexo & & & & \\
$\quad$ Hembra & 60 & $23.48 \pm 2.59^{\text {a }}$ & 29.67 & 18.24 \\
$\quad$ Macho & 60 & $23.23 \pm 2.74^{\text {a }}$ & 29.22 & 18.12 \\
Comunidad & & & & \\
$\quad$ Condormilla Bajo & 60 & $23.27 \pm 2.53^{\text {a }}$ & 29.15 & 18.54 \\
$\quad$ Condormilla Alto & 60 & $23.44 \pm 2.80^{\text {a }}$ & 29.67 & 18.12 \\
\hline
\end{tabular}

$a, b, c$ Superíndices diferentes dentro de columnas y variables indica diferencia significativa $(p<0.05)$

Cuadro 2. Factor de confort (\%) de fibra en alpacas Huacaya, según edad, sexo y procedencia

\begin{tabular}{lcccc}
\hline Variable & N. $^{\circ}$ & Media \pm D.E. & Máximo & Mínimo \\
\hline Edad (años) & & & & \\
2 & 40 & $95.34 \pm 2.84^{\mathrm{a}}$ & 98.73 & 85.98 \\
4 & 40 & $92.99 \pm 4.21^{\mathrm{b}}$ & 98.30 & 83.38 \\
6 & 40 & $90.22 \pm 3.98^{\mathrm{c}}$ & 96.89 & 80.63 \\
Sexo & & & \\
$\quad$ Hembra & 60 & $92.83 \pm 4.32^{\mathrm{a}}$ & 98.73 & 80.90 \\
$\quad$ Macho & 60 & $92.87 \pm 4.21^{\mathrm{a}}$ & 98.30 & 80.63 \\
Comunidad & & & \\
$\quad$ Condormilla Bajo & 60 & $93.14 \pm 4.22^{\mathrm{a}}$ & 98.50 & 80.90 \\
$\quad$ Condormilla Alto & 60 & $92.56 \pm 4.30^{\mathrm{a}}$ & 98.73 & 80.63 \\
\hline
\end{tabular}

$a, b, c$ Superíndices diferentes dentro de columnas y variables indica diferencia significativa $(p<0.05)$

encontraron valores de factor de confort de $93.67 \%$, el cual se considera como un buen valor acorde a los requerimientos de la industria textil.

Ormachea et al. (2015) reporta $96.19 \%$ de factor de confort en hembras y $94.99 \%$ en machos, indicando que los vellones de las alpacas hembras brindan un mayor factor de confort debido al menor diámetro de fibra en comparación a los machos. Por otro lado, los resultados encontrados son superiores a lo reportado por Lupton y Stobart (2006) quienes obtuvieron diámetros de fibra de $26.7 \mu \mathrm{m}$ y un factor de confort de $73 \%$ en hembras en comparación al $27.1 \mu \mathrm{m}$ y $70.6 \%$ en machos.

Con respecto a la comunidad, Quispe (2010) reportó que existe suficiente evidencia del efecto de la comunidad sobre el factor de confort; sin embargo, se dispone de 
Cuadro 3. Índice de curvatura ( $\mathrm{grad} / \mathrm{mm})$ de fibra en alpacas Huacaya, según edad, sexo y procedencia

\begin{tabular}{lcccc}
\hline Variable & N. $^{\circ}$ & Media \pm D.E. & Máximo & Mínimo \\
\hline Edad (años) & & & & \\
2 & 40 & $38.35 \pm 4.18^{\text {a }}$ & 47.58 & 31.18 \\
4 & 40 & $34.95 \pm 3.71^{\text {a }}$ & 44.33 & 26.38 \\
6 & 40 & $31.74 \pm 4.47^{\text {a }}$ & 39.82 & 24.33 \\
Sexo & & & \\
$\quad$ Hembra & 60 & $34.80 \pm 4.80^{\text {a }}$ & 47.58 & 24.33 \\
$\quad$ Macho & 60 & $35.23 \pm 5.05^{\text {a }}$ & 45.60 & 24.33 \\
Comunidad & & & \\
$\quad$ Condormilla Bajo & 60 & $35.38 \pm 4.79^{\text {a }}$ & 47.58 & 24.33 \\
$\quad$ Condormilla Alto & 60 & $34.65 \pm 5.04^{\text {a }}$ & 44.33 & 24.33 \\
\hline
\end{tabular}

${ }^{\mathrm{b}}$ Superíndices iguales dentro de columnas y variables indican ausencia de diferencia significativa

información que contradicen estas afirmaciones. Por ejemplo, Ormachea et al. (2015) reporta valores de 95.62 y $95.56 \%$ en dos cominades del distrito de Corani, Puno, y Diaz et al. (2015) reporta valores de 97.43, 97.19 y $96.88 \%$ en alpacas de tres comunidades del distrito de Macusani, Perú, en ambos casos, sin diferencias significativas entre comunidades, probablemente debido a la presión de selección para menor diámetro de fibra y alto porcentaje de factor de confort.

\section{Índice de Curvatura}

El índice de curvatura de la fibra no fue afectado por la edad, sexo o procedencia (Cuadro 3). Los resultados obtenidos fueron superiores a los valores de $38.8 \mathrm{grad} / \mathrm{mm}$ reportados por Quispe (2010), mientras que Marín (2007) encuentra valores de 47.14 $\mathrm{grad} / \mathrm{mm}$ en alpacas de un año. La diferencia obtenida probablemente se debe al tamaño de muestra utilizado y a la categoría del animal. Al respecto, se conoce que fibras con alta curvatura tienen un menor diámetro (Fish et al., 1999; Mamani, 2009). Por otro lado, Ormachea et al. (2015) obtuvo valores de $43.43,42.21$ y $41.27 \mathrm{grad} / \mathrm{mm}$ en alpacas
Huacaya 2, 3 y 4 años, respectivamente, sin diferencias significativas entre grupos etarios.

El factor sexo no tuvo influencia en el índice de curvatura, lo cual ha sido reportado en la literatura (por Lupton y Stobart, 2006; Siguayro, 2010; Ormachea et al., 2015). Con respecto al factor comunidad, los resultados concuerdan con el estudio de Ormachea et al. (2015), quienes reportan valores de 42.44 \pm 6.71 y $42.16 \pm 6.22 \mathrm{grad} / \mathrm{mm}$ en alpacas Huacaya de color blanco de dos comunidades del distrito de Corani, Puno.

\section{Finura al Hilado}

La finura al hilado se afectó significativamente con la edad $(\mathrm{p}<0.05)$, no habiendo diferencia por efecto del sexo o procedencia (Cuadro 4). Quispe (2010) encontró en alpacas Huacaya de color blanco una finura al hilado de $20.9 \mu \mathrm{m}$, observando que los animales jóvenes tienen menores valores que animales adultos, siendo los animales menores de 18 meses los que exhiben una mejor finura al hilado. Por otro lado, Vásquez et al. (2015) reporta para alpacas de las zonas alto andinas de Apurímac valores de 17.4, 19.2, 
Cuadro 4. Finura al hilado $(\mu \mathrm{m})$ de fibra en alpacas Huacaya, según edad, sexo y procedencia

\begin{tabular}{lcccc}
\hline Variable & N. $^{\circ}$ & Media \pm D.E. & Máximo & Mínimo \\
\hline Edad (años) & & & & \\
2 & 40 & $21.7 \pm 2.1^{\text {a }}$ & 28.69 & 18.10 \\
4 & 40 & $23.8 \pm 2.1^{\mathrm{b}}$ & 28.99 & 20.10 \\
6 & 40 & $25.4 \pm 2.2^{\mathrm{c}}$ & 29.34 & 21.07 \\
Sexo & & & \\
$\quad$ Hembra & 60 & $23.9 \pm 2.6^{\mathrm{a}}$ & 18.10 & 29.34 \\
$\quad$ Macho & 60 & $23.4 \pm 2.6^{\mathrm{a}}$ & 18.36 & 29.34 \\
Comunidad & & & \\
$\quad$ Condormilla Bajo & 60 & $23.7 \pm 2.7^{\text {a }}$ & 18.10 & 29.34 \\
$\quad$ Condormilla Alto & 60 & $23.7 \pm 2.4^{\text {a }}$ & 18.36 & 29.34 \\
\hline a,b,c Superíndices diferentes dentro de columnas &
\end{tabular}

$a, b, c$ Superíndices diferentes dentro de columnas y variables indica diferencia significativa $(p<0.05)$

Cuadro 5. Longitud de mecha $(\mathrm{cm})$ de fibra en alpacas Huacaya, según edad, sexo y procedencia

\begin{tabular}{lcccc}
\hline Variable & N. & Media \pm D.E. & Máximo & Mínimo \\
\hline Edad (años) & & & & \\
2 & 40 & $13.7 \pm 1.9^{\mathrm{a}}$ & 18.5 & 11.5 \\
4 & 40 & $10.2 \pm 1.1^{\mathrm{b}}$ & 13.4 & 8.5 \\
6 & 40 & $8.8 \pm 1.0^{\mathrm{c}}$ & 10.5 & 6.5 \\
Sexo & & & \\
$\quad$ Hembra & 60 & $10.8 \pm 2.4^{\mathrm{a}}$ & 17.5 & 6.5 \\
$\quad \begin{array}{l}\text { Macho } \\
\text { Comunidad }\end{array}$ & 60 & $10.6 \pm 2.5^{\mathrm{a}}$ & 18.5 & 7.5 \\
$\quad$ Condormilla Bajo & 60 & $10.7 \pm 2.2^{\mathrm{a}}$ & 18.5 & 7.5 \\
$\quad$ Condormilla Alto & 60 & $10.7 \pm 2.6^{\mathrm{a}}$ & 17.5 & 6.5 \\
\hline
\end{tabular}

a,b,c Superíndices diferentes dentro de columnas y variables indica diferencia significativa $(p<0.05)$

Cuadro 6. Correlaciones entre el diámetro de la fibra y características textiles de la fibra de alpacas Huacaya

\begin{tabular}{lcccc}
\hline & Finura al hilado & $\begin{array}{c}\text { Índice de } \\
\text { curvatura }\end{array}$ & $\begin{array}{c}\text { Factor de } \\
\text { confort }\end{array}$ & $\begin{array}{c}\text { Longitud de } \\
\text { mecha }\end{array}$ \\
\hline $\begin{array}{l}\text { Diámetro de } \\
\text { fibra }\end{array}$ & $0.7500^{*}$ & $-0.4019^{*}$ & $-0.57614^{*}$ & $-0.08157^{\text {n.s. }}$ \\
\hline
\end{tabular}

*: $p<0.05$ : n.s: no significativo 
20.2 y $21.6 \mu \mathrm{m}$ en alpacas diente de leche, dos dientes, cuatro dientes y boca llena, respectivamente, en comunidades de las zonas altoandinas de Apurímac, Perú. El mayor valor encontrado en el presente estudio pudo deberse al diámetro de fibra y al coeficiente de variación (Ormachea, 2012).

Con respecto al sexo, los resultados obtenidos se asemejan con lo mencionado por Diaz et al. (2015), quienes manifiestan que el lugar de procedencia no tiene efecto en la finura al hilado.

\section{Longitud de Mecha}

La longitud de mecha disminuyó significativamente con la edad $(p<0.05)$, no habiendo diferencia por efecto del sexo o procedencia (Cuadro 5).

Los resultados del presente estudio siguen la misma tendencia de los estudios de Loza (2000), quien encontró una menor longitud de fibra en la segunda esquila (10.9 \pm $1.1 \mathrm{~cm}$ ) en comparación con la primera esquila $(11.4 \pm 1.6 \mathrm{~cm})$, y con Pinazo (2000), quién reporta $12.7 \mathrm{~cm}$ para el primer año de edad, para luego ir decreciendo hasta el sexto año con $10.15 \mathrm{~cm}$. Por otro lado, Mamani (2009) obtuvieron una longitud de mecha mayor para alpacas de 2 dientes $(12.3 \mathrm{~cm})$ que en alpacas con dientes de leche (9.97 $\mathrm{cm}$ ), debido a que, en las comunidades en estudio, las alpacas son esquiladas a más de un año y, por ello, tienen una mayor longitud de mecha.

Pinazo (2000) encontró promedios de longitud de mecha de $9.16 \mathrm{~cm}$ para machos y de $9.58 \mathrm{~cm}$ para las hembras, no encontrando diferencia entre sexos, al igual que en el presente estudio. Asimismo, Bustinza (2001) indica que la longitud de mecha en alpacas de ambos sexos no presentan diferencias estadísticas. No obstante, Flores (2006) reporta promedios superiores en hembras (15.39 $\mathrm{cm})$ que en machos $(11.67 \mathrm{~cm})$ mientras que autores como Montesinos (2000) encuentran mayor longitud de mecha en machos (12.15 $\mathrm{cm})$ que en hembras $(11.81 \mathrm{~cm})$, al igual que Loza (2000): machos $(11.51 \mathrm{~cm})$ y hembras $(10.79 \mathrm{~cm})$

\section{Correlaciones entre Características Pro- ductivas y Textiles de la Fibra}

Se encontró una correlación negativa y moderada para las variables de diámetro de fibra con el índice de curvatura (-0.04) y factor de confort (-0.58), lo cual concuerda con lo indicado por diversos autores (Fish et al., 1999; Holt, 2006; Ormachea, 2013; Diaz et al., 2015), quienes manifiestan que a menor diámetro de fibra el valor del factor de confort e índice de curvatura será mayor.

Holt (2006) determinó, por otro lado, coeficientes de correlación de intensidad media entre el diámetro de fibra y el índice de curvatura (-0.64), Siguayro (2010) indicó correlaciones entre estos parámetros de -0.20 para machos y de -0.14 para hembras, Marín (2007) encontró correlaciones en alpacas Huacaya de un año que oscilan entre $-0.35 \mathrm{y}$ -0.70 , relativamente similar a los resultados de McGregor (2006) quien reportó una relación negativa (-0.16) en el sur de Australia. Asimismo, Lupton y Stobart (2006) indican una relación negativa alta (-0.86) en EEUU, donde las fibras con alta curvatura tenían menor diámetro.

La relación del diámetro de fibra con la finura al hilado mostró una correlación positiva de intensidad alta (0.75). Al respecto, Vásquez et al. (2015), reporta una correlación positiva (0.99).

\section{Conclusiones}

- El diámetro de fibra se incrementó y la finura al hilado se afectó significativamente con la edad, en tanto que el índice de confort y la longitud de mecha disminuyen significativamente con la edad del animal. 
- El índice de curvatura no se afecta con la edad, sexo o procedencia del animal.

- El sexo y la comunidad de procedencia no afectaron el diámetro de fibra el índice de confort, la longitud de mecha ni la finura al hilado.

- Existe una correlación negativa y moderada entre el diámetro de fibra con el índice de curvatura $(\mathrm{r}=-0.40)$ y factor de confort $(r=-0.58)$, en tanto que se observa una correlación alta entre diámetro de fibra y finura al hilado $(\mathrm{r}=0.75)$.

\section{Literatura Citada}

1. Aylan-Parker J, McGregor B. 2002. Optimising sampling techniques and estimating sampling variance of fleece quality attributes in alpacas. Small Ruminant Res 44: 53-64. doi: 10.1016/ S0921-4488(02)00038-X

2. ASTM. 1999. Standard test method of sampling and testing stage length of grease wool. In: Annual Book of ASTM Standards, Designation: D 1234-85 (Reapproved 2001). Vol 07(01). Philadelphia: ASTM. p 1-4.

3. Benavides M, Vasquez G, Casafranca $J$. 1996. La pequeña agroindustria en el Perú, situación actual y perspectiva. Lima, Perú: Prodar. 87 p.

4. Brims M, Peterson A, Gherardi S. 1999. Introducing the OFDA2000 - For rapid measurement of diameter profile on greasy wool staples. IWTO, Raw Wool Group Rep. RWG04, Florence, Italy.

5. Bustínza AV. 2001. La alpaca conocimiento del gran potencial andino. Libro 1. Puno, Perú: Univ. Nacional del Altiplano. $495 \mathrm{p}$.

6. Cordero F, Contreras P, Mayhua M, Jurado E, Castrejón V. 2011. Correlaciones fenotípicas entre características productivas en alpacas Huacaya. Rev Inv Vet Perú 22: 15-21. doi: /10.15381/ rivep.v22i1.114
7. Diaz J, Alencastre R, Ormachea E, Catacora N. 2015. Determinación de las principales características de la fibra de alpaca Huacaya y Suri en la Provincia de Carabaya - Sector Chocoaquilla. En: VII Congreso Mundial de Camelidos Sudamericanos. Puno, Perú.

8. [FAO] Organización de las Naciones Unidas para la Alimentación y la Agricultura. 2005. Situación actual de los camélidos sudamericanos en el Perú. Proyecto de cooperación técnica en apoyo a la crianza y aprovechamiento de los camélidos sudamericanos en la Región Andina. Lima, Perú: FAO. 62 p.

9. Fish VE, Mahar TJ, Crook BJ. 1999. Fibre curvature morphometry and measurement. Wool Technol Sheep Breeding 47: 248-265.

10. Flores A. 2006. Determinación del diámetro de fibra y longitud de mecha en alpacas (Vicugna pacos) de la provincia de Tarata - Tacna. Tesis de Médico Veterinario y Zootecnista. Tacna, Perú: Univ. Nacional Jorge Basadre Grohmann. $81 \mathrm{p}$.

11. Franco F, San Martín H, Ara G, Olazábal L, Carcelén C. 2009. Efecto del nivel alimenticio sobre el rendimiento y calidad de fibra en alpacas. Rev Inv Vet Perú 20: 187-195. doi: 10.15381/ rivep.v20i2.605

12. Holt C. 2006. A survey of the relationships of crimp frequency, micron, character and fibre curvature. A report to the Australian Alpaca Association. Pambula Beach NSW, Australia. 33 p.

13. Huamaní R, Gonzáles C. 2004. Efecto de la edad y el sexo en los parámetros físicos de la fibra de alpaca (Lama pacos) Huacaya en Huancavelica. Tesis de Ingeniero Zootecnista. Huanca-velica: Univ. Nacional de Huancavelica. $80 \mathrm{p}$.

14. Huanca T, Apaza N, Lazo A. 2007. Evaluación del diámetro de fibra en alpacas de las comunidades de los distritos de Cojata y Santa Rosa-Puno. Arch Latinoamer Prod Anim 15: 480.

15. [INEI] Instituto Nacional de Estadística e Informática. 2012. IV Censo Nacional Agropecuario 2012. [Internet]. 
Disponible en: http://censos.inei.gob.pe/ cenagro/tabulados/

16. Loza J. 2000. Características físicas de la fibra de la alpaca Huacaya de color del C.I.P. La Raya. Tesis de Médico Veterinario Zootecnista. Puno, Perú: Univ. Nacional del Altiplano. $82 \mathrm{p}$.

17. Lupton CAM, Stobart R. 2006. Fiber characteristics of the Huacaya alpaca. Small Ruminant Res 64: 211-224. doi: 10.1016/j.smallrumres.2005.04.023

18. Machaca VM, Choque AB, Arizapana FC, Ocsa VP, Peña EQ, Machaca RM. 2017. Características de la fibra de alpaca Huacaya de Cotaruse, Apurímac, Perú. Rev Inv Vet Perú 28: 843-851. doi: 10.15381/rivep.v28i4.13889

19. Mamani A. 2009. Correlación entre el diámetro, densidad y rizo de la fibra de alpaca Huacaya hembra, según región corporal. Tesis de Médico Veterinario y Zootecnista. Puno, Perú: Univ. Nacional del Altiplano. $89 \mathrm{p}$.

20. Marín E. 2007. Efecto del sexo sobre las características tecnológicas y productivas en alpacas tuis para su uso en la industria textil. Tesis de Maestría. Lima, Perú: Univ. Nacional Agraria La Molina. $76 \mathrm{p}$.

21. McGregor BA. 2006. Production attributes and relative value of alpaca fleeces in southern Australia and implications for industry development. Small Ruminant Res 61: 93-111. doi: 10.1016/j.smallrumres.2005.07.001

22. McGregor BA, Butler K. 2004. Sources of variation in fiber diameter attributes of Australian alpacas and implications for fleece evaluation and attributes of Australian alpacas and implications for fleece evaluation and animal selection. Aust J Agr Res 55: 433442. doi: 10.1071/AR03073

23. Montes M, Quicaño I, Quispe R, Quispe E, Alfonso L. 2008. Quality characteristics of Huacaya alpaca fibre produced in the Peruvian Andean Plateau region of Huancavelica. Span J Agric Res 6:33-38. doi: 10.5424/sjar/2008061-5258
24. Montesinos R. 2000. Características físicas de la fibra de alpacas Huacaya y Suri de color en el banco de germoplasma Quimsachata, ILLPA-INIA-Puno. Tesis de Médico Veterinario Zootecnista: Univ. Nacional del Altiplano. $89 \mathrm{p}$.

25. Morante R, Goyache F, Burgos A, Cervantes I, Péres-Cabal $M$, Gutiérrez J. 2009. Genetic improvemente for alpaca fibre production in the Peruvian Altiplano: the Pacomarca experience. Anim Genet Resour Informat 45: 37-43. doi: 10.1017/ S1014233909990307

26. Ormachea E. 2012. Características de la fibra de alpaca analizadas con el método OFDA 2000. ALLPAK'A 16: 83-91.

27. Ormachea E. 2013. Diámetro de fibra, factor de confort e índice de curvatura en alpacas Huacaya de las comunidades de Quelccaya y Chimboya del distrito de Corani - Carabaya - Puno. Tesis de Médico Veterinario Zootecnista. Puno: Perú: Univ. Nacional del Altiplano. $78 \mathrm{p}$.

28. Ormachea E, Calsín B, Olarte U. 2015. Características textiles de la fibra en alpacas Huacaya del distrito de Corani Carabaya, Puno. Rev Invest Alto Andinas 17: 215-220. doi: 10.18271/ ria.2015.115

29. Pinazo R. 2000. Algunas características físicas de la fibra de alpaca Huacaya y Suri del C.E. La Raya. Tesis de Médico Veterinario Zootecnista: Univ. Nacional del Altiplano. $68 \mathrm{p}$

30. Quispe E. 2010. Evaluación de características productivas y textiles de la fibra de alpacas Huacaya de la región de Huancavelica, Perú: Simposio Internacional de Fibras de Camélidos Sudamericanos. Huancavelica, Perú.

31. Quispe E, Paucar R, Poma A, Sachero $D$, Mueller J. 2008. Perfil de diámetro de fibra en alpacas, Seminario Internacional de Biotecnología Aplicada en Camélidos Sudamericanos. Huancavelica, Perú: Univ. Nacional de Huancavelica. 
32. Quispe E, Alfonso L, Flores A, Guillen $H$, Ramos Y. 2009a. Bases to an improvement program of the alpacas in highland region at Huancavelica-Perú. Arch Zootec 58: 705-716.

33. Quispe EC, Rodríguez, TC, Iñiguez LR, Mueller JP. 2009b. Producción de fibra de alpaca, llama, vicuña y guanaco en Sudamérica. Recursos Genéticos Animales 45: 1-14. doi: 10.1017/S1014233909990277

34. $R$ Core Team. 2014. R: a language andenvironment for statistical computing. $\mathrm{R}$ Foundation for Statistical Computing.Vienna, Austria. [Internet]. Available in: http://www.R-project.org

35. Siguayro R. 2010. Comparación de las características físicas de las fibras de la llama ch'aku (Lama glama) y la alpaca Huacaya (Lama pacos) del centro experimental Quimsachata del INIA-Puno.
Tesis de Maestría. Lima, Perú: Univ. Nacional Agraria La Molina. $63 \mathrm{p}$.

36. Vásquez O, Gómez OE, Quispe E.2015. Características tecnológicas de la fibra blanca de alpaca Huacaya en la zona altoandina de Apurímac. Rev Inv Vet Perú 26: 213-222. doi: 10.15381/ rivep.v26i2.11020

37. Vilcanqui H. 2008. Efecto de la edad y el sexo sobre las características tecnológicas de la fibra de vicuña en la provincia de Castrovirreyna-Huancavelica. Tesis de Magíster. Lima, Perú: Univ. Nacional Agraria La Molina. Lima. $85 \mathrm{p}$.

38. Wuliji T, David G, Dodds K, Turner R, Andrews R, Bruce G. 2000. Production performance, repeat ability and heritability estimates for live weight, fleece weight and fiber characteristics of alpaca in New Zeland. Small Ruminant Res 37: 189-201. doi: 10.1016/ S0921-4488(00)00127-9 